BMC

Physiology

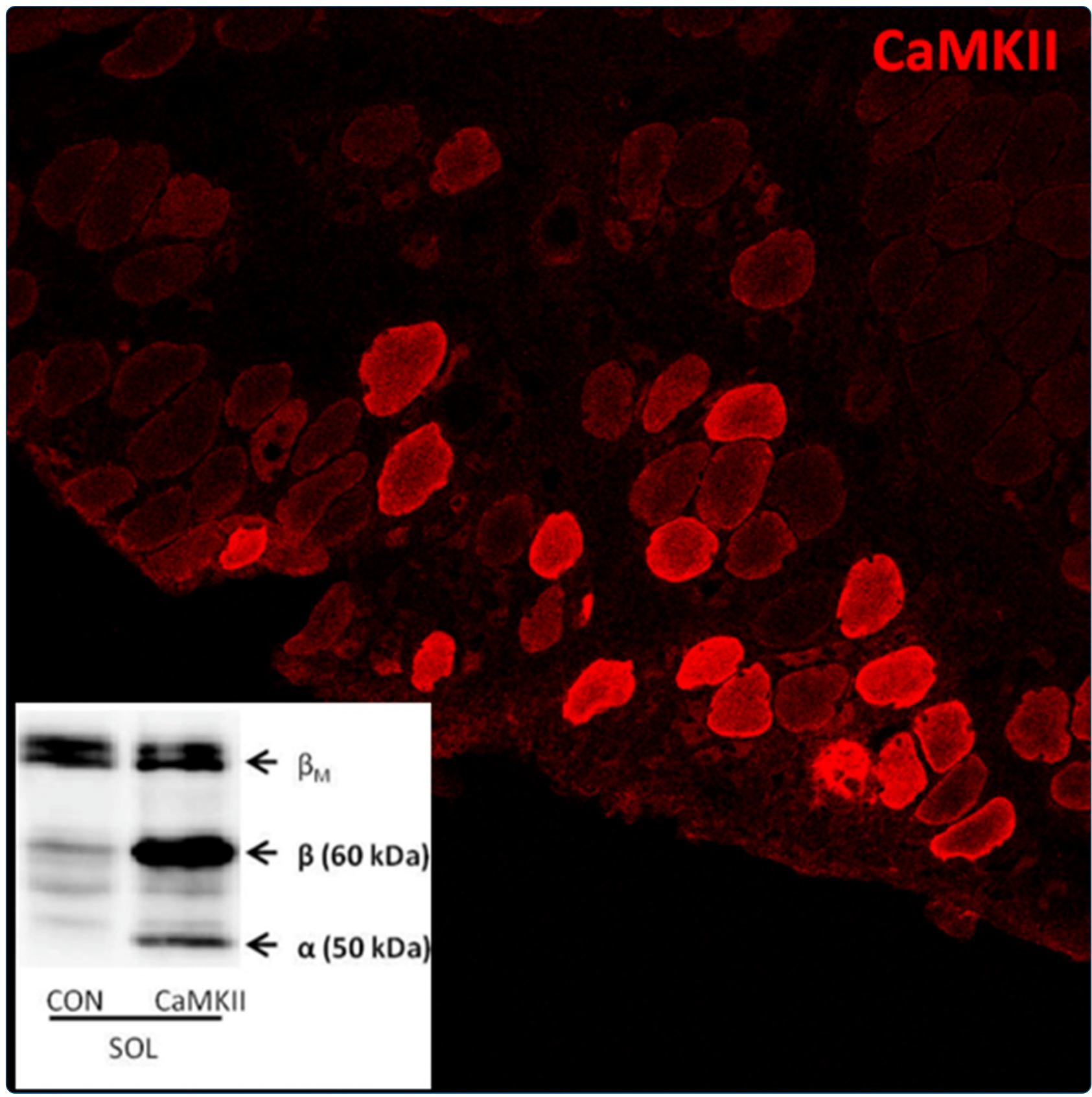

CaMKII content affects contractile, but not mitochondrial, characteristics in regenerating skeletal muscle

Eilers et al. 


\title{
CaMKII content affects contractile, but not mitochondrial, characteristics in regenerating skeletal muscle
}

\author{
Wouter Eilers ${ }^{1}$, Richard T Jaspers ${ }^{2}$, Arnold de Haan ${ }^{1,2}$, Cline Ferri ${ }^{3}$, Paola Valdivieso ${ }^{3}$ and Martin Flck ${ }^{1,3^{*}}$
}

\begin{abstract}
Background: The multi-meric calcium/calmodulin-dependent protein kinase II (CaMKII) is the main CaMK in skeletal muscle and its expression increases with endurance training. CaMK family members are implicated in contraction-induced regulation of calcium handling, fast myosin type IIA expression and mitochondrial biogenesis. The objective of this study was to investigate the role of an increased CaMKII content for the expression of the contractile and mitochondrial phenotype in vivo. Towards this end we attempted to co-express alpha- and beta-CaMKII isoforms in skeletal muscle and characterised the effect on the contractile and mitochondrial phenotype.

Results: Fast-twitch muscle m. gastrocnemius (GM) and slow-twitch muscle $m$. soleus (SOL) of the right leg of 3-month old rats were transfected via electro-transfer of injected expression plasmids for native $\alpha / \beta$ CaMKII. Effects were identified from the comparison to control-transfected muscles of the contralateral leg and non-transfected muscles. $\alpha / \beta$ CaMKII content in muscle fibres was 4-5-fold increased 7 days after transfection. The transfection rate was more pronounced in SOL than GM muscle (i.e. 12.6 vs. 3.5\%). The overexpressed a/ $\beta$ CaMKII was functional as shown through increased threonine 287 phosphorylation of $\beta$-CaMKII after isometric exercise and down-regulated transcripts COXI, COXIV, SDHB after high-intensity exercise in situ. a/ $\beta$ CaMKIl overexpression under normal cage activity accelerated excitation-contraction coupling and relaxation in SOL muscle in association with increased SERCA2, ANXV and fast myosin type IIA/X content but did not affect mitochondrial protein content. These effects were observed on a background of regenerating muscle fibres.

Conclusion: Elevated CaMKII content promotes a slow-to-fast type fibre shift in regenerating muscle but is not sufficient to stimulate mitochondrial biogenesis in the absence of an endurance stimulus.
\end{abstract}

Keywords: CaMKII, Skeletal muscle, Plasticity, Excitation, Contraction, Mitochondria

\section{Background}

Repeated muscle contractions rely on motoneuron-driven variations in sarcoplasmic calcium though ryanodine receptor-mediated release of calcium from the sarcoplasmic reticulum (SR) and the subsequent re-uptake of calcium via SERCA channels [1]. The observed magnitude of the rise in sarcoplasmic calcium varies between slow and fast muscle types, which suggests it may control the

\footnotetext{
* Correspondence: mflueck@research.balgrist.ch

${ }^{1}$ Institute for Biomedical Research into Human Movement and Health, Manchester Metropolitan University, John Dalton Building, Oxford Road, M1 5GD Manchester, United Kingdom

${ }^{3}$ Laboratory for Muscle Plasticity, Department of Orthopaedics, University of Zurich, Balgrist University Hospital, Forchstrasse 340, 8008 Zurich, Switzerland Full list of author information is available at the end of the article
}

contractile characteristics of muscle fibres [2]. However, this relationship is not fixed and muscle fibre makeup in SR and contractile proteins demonstrates a certain degree of plasticity in response to contractile stimuli [3]. An important feature of repeated muscle work (i.e. endurance training) is the specific increase in mitochondrial content; reflecting a compensatory strategy to meet the energy demand of fibres undergoing frequent rounds of actin and myosin cross-bridge cycling [4]. In the rat, the mitochondrial adaptations of exercised skeletal muscle are associated with a transition of myosin isoform expression towards a slow-twitch phenotype [5,6]. The observed transformation of muscle fibre types is associated with a chronic rise in sarcoplasmic calcium [7-9], which implicates 
calcium-dependent biochemical pathways in the regulation of muscle plasticity.

The calcium/calmodulin-dependent phosphatase calcineurin and calcium/calmodulin-dependent kinases (CaMK) are important transducers of calcium signals towards gene expression $[2,10]$. The calcineurin-mediated pathway has been shown to regulate expression of slow fibre type-related myofibrillar proteins [11-13] and affects mitochondrial gene expression although calcineurin does not appear required for exercise-induced mitochondrial biogenesis [14-16]. By contrast, CaMK activation has been implied to regulate mitochondrial biogenesis $[9,17,18]$, type IIA myosin heavy chain (MHCIIA) expression $[19,20]$ and calcium re-uptake into the SR in slow type muscle fibres [21].

However, the current understanding of the physiological role of CaMK holoenzymes in whole muscle is incomplete, as previous studies rely on short-term inhibition studies with pharmacological agents in single muscle fibres [22] or the overexpression of a CaMKIV mutant which is not expressed in skeletal muscle [23] and has lost its calcium-dependent regulation [18]. CaMKII is the main CaMK isoform in skeletal muscle. Importantly, however, the effects of CaMKII on the expression of genes that underlie muscle plasticity have - apart from the study of the glucose transporter GLUT4 [24] - not been addressed.

CaMKII operates as a hetero-meric phospho-transferase which can decode calcium transients through autophosphorylation at threonine-287 ([2,25,26]). Threonine-287 phosphorylation of CaMKII is increased after acute exercise in rats and humans $[27,28]$, indicating that CaMKII is part of the signalling pathways integrating the effects of exercise on muscle structure and function (reviewed by $[2,29]$ ). These findings emphasize that CaMKII activation is firmly associated with the regulation of the oxidative muscle phenotype with contractile paradigms $[2,30]$. This is corroborated by the concomitant increase in CaMKII and mitochondrial ATP synthase expression with endurance exercise training [31,32]. However, the question remains to which extent contractile features, as shown by CaMK inhibition in vitro $[19,20]$, depend on CaMKII in vivo, and to which extent downstream effects of CaMKII-mediated calcium sensing would be differently affected between contractile muscle phenotypes [33,34].

We hypothesized that the content of hetero-multimeric CaMKII controls the mitochondrial and contractile phenotype of skeletal muscle and that this would take place in both slow-twitch $m$. soleus (SOL) and fast-twitch m. gastrocnemius (GM). We tested this assumption by assessing the effects of the co-overexpression of native alpha- and beta-CaMKII isoforms, with similar substrate specificity and structure as the skeletal muscle CaMKII isoforms [35,36] on selected protein markers of the contractile and mitochondrial phenotype. Additionally, the corresponding transcript response to high-intensity exercise in situ was measured along with functional characteristics of the targeted SOL and GM muscles. Because the muscles under investigation show fibre recruitment during self-initiated locomotion [37] we assumed that the effect of CaMKII overexpression would manifest under normal cage activity without an imposed contraction protocol. Control experiments were carried out to quantify the extent of muscle regeneration being associated with the selected electro-transfer method to produce overexpression from injected expression plasmids [38-40].

\section{Results}

CaMKII overexpression and phosphorylation in skeletal muscle

$\alpha / \beta$-CaMKII-transfection increased protein levels of $\alpha$ - and $\beta$-CaMKII isoforms at 50 and $60 \mathrm{kDa}$, respectively, compared to control-transfected SOL muscle (Figure 1A). In vitro experiments demonstrated $\mathrm{Ca}^{2+} / \mathrm{CaM}$-dependent phosphorylation of the introduced $\beta$ CaMKII (Figure 1C). Overexpression of $\alpha / \beta$ CaMKII could be detected in $12.6 \%$ of fibres in SOL muscle and at a lower level, i.e. $3.5 \%$, in GM muscle ( $\mathrm{p}=0.002)$. $\alpha$ - and $\beta$-CaMKII content was increased at the level of total protein in SOL muscle (Figure 1D/E). Fibres in SOL and GM muscle which demonstrated elevated CaMKII expression after the transfection of $\alpha / \beta$ CaMKII plasmid had 5.30 .8 and 4.00 .5 fold increased CaMKII levels, respectively. Mean cross sectional area (MCSA) of the transfected muscle fibres was lower than the non-transfected fibres in the $\alpha / \beta$ CaMKII-transfected SOL muscle (transfected vs. non-transfected muscle fibre: 2675132 vs. 3706 $86 \mu \mathrm{m} 2 ; \mathrm{p}=0.001)$.

\section{$a$-and $\beta$-CaMKII co-overexpression shifts gene expression towards a fast phenotype with enhanced calcium handling}

Factorial analysis of the paired data revealed a main effect $(\mathrm{p}<0.05)$ of the co-overexpression of the native $\alpha$ - and $\beta$-CaMKII isoforms on the contractile parameters time-to-peak-twitch force $(-16 \%)$ and half-relaxation-time $(-15 \%)$ for the combined data from SOL and GM muscle (Figure 2). In SOL muscle alone, a trend for a shortened half-relaxation-time was identified. Maximum twitch force and maximum tetanic force, and fatigability of tetanic force, did not differ between control- and $\alpha / \beta$ CaMKII-transfected GM and SOL.

We assessed the effect of $\alpha / \beta$ CaMKII overexpression on the levels of proteins associated with the acceleration of muscle contraction (i.e. MHCIIA/IIX and IIB) and relaxation (SERCA2, ANXV; [4, 67]). This analysis was limited to SOL, because of the higher transfection efficiency 

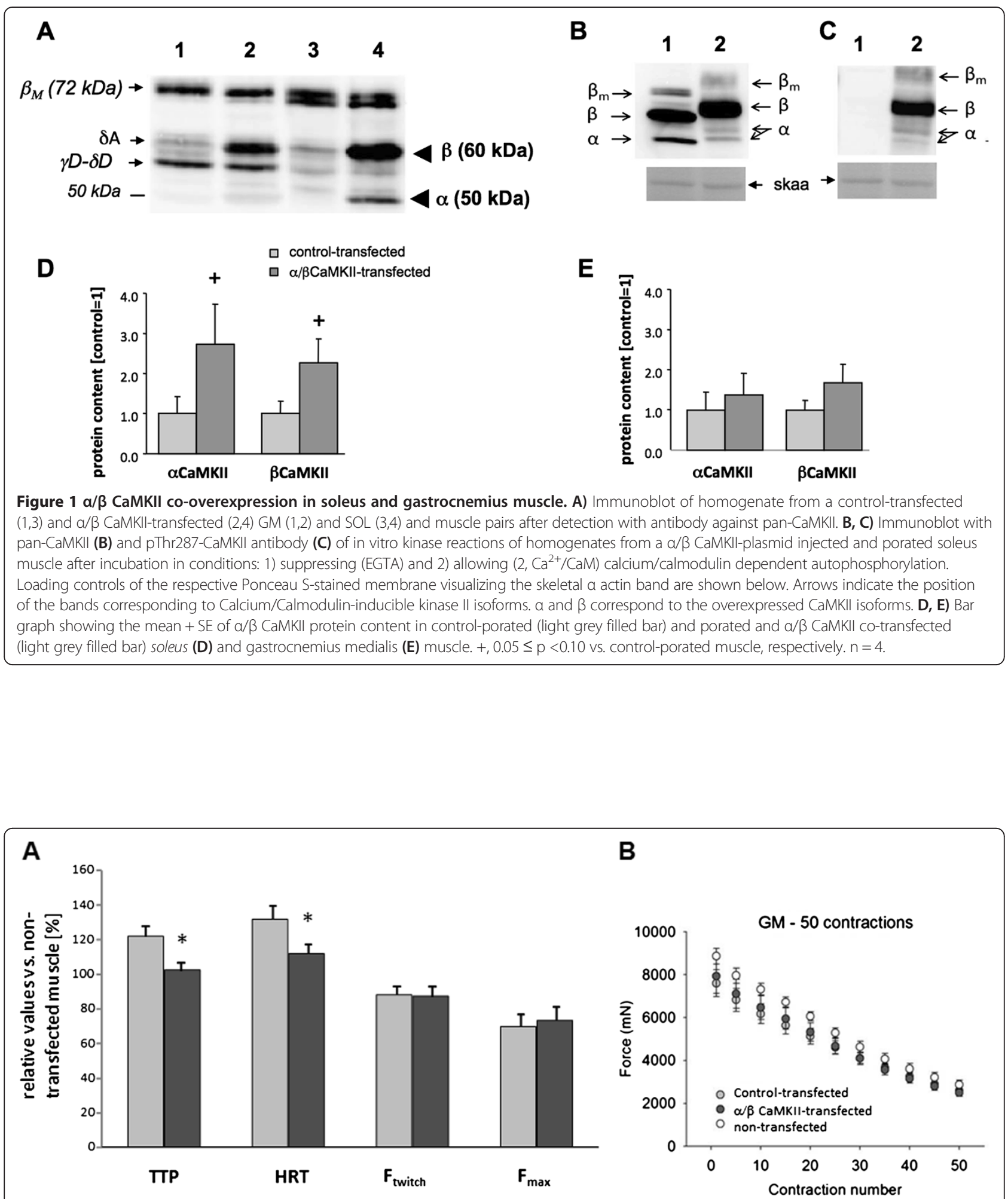

Figure 2 Contractile effects of $\boldsymbol{\alpha} / \boldsymbol{\beta}$ CaMKII co-overexpression. A) Mean + SE of values for the contractile parameters time-to-peak-twitch force (TTP), half-relaxation time $(H R T)$, maximum twitch force $\left(F_{\text {twitch }}\right)$ and maximum tetanic force $\left(F_{\max }\right)$ for the SOL and GM muscle combined being subjected to control-transfection (light grey filled bar) or a/ $\beta$ CaMKIl-transfection (dark grey filled bar). Values reflect values relative to the average seen in non-transfected muscle $(n=7)$. ${ }^{*}, p \leq 0.05$ vs. control-transfected muscle. Repeated ANOVA with post hoc test of Fisher $(n=11)$. B) Scatter plot of mean + SE of force values of fifty paced tetanic contractions in non-transfected, control-transfected, and a/B CaMKIl-transfected GM muscle. $n=6-9$ per group. 
achieved in this muscle. We found that the expression of all three proteins, myosin heavy chain isoforms IIA/IIX, SERCA2 and ANXV, was increased in SOL muscle compared to contra-lateral control-transfected SOL muscle (Figures 3 and 4).

Examples of the immunofluorescence detection of CaMKII, MHCI and MHCII are shown in Figure 4A-C. A larger fraction of CaMKII-transfected fibres expressed MHCII than non-transfected fibres ( 0.36 vs. $0.18 ; \mathrm{p}=0.0008$, Chi2-Test). Quantification of the co-staining of CaMKII and SERCA2 (Figure 4D/E) revealed a significantly higher staining intensity of SERCA2 in CaMKII-overexpressing SOL fibres compared to that in non-transfected fibres of the same muscle (Figure 4F).

Protein levels for constituents of the oxidative phosphorylation chain were not affected by $\alpha / \beta$ CaMKII overexpression (Figure 5A). We assessed whether overexpression of $\alpha / \beta$ CaMKII was sufficient to increase COXIV protein expression at the fibre level (Figure 5C). COXIV staining intensity in transfected muscles did not differ between CaMKII-overexpressing and non-transfected fibres in SOL muscle (Figure 5D).

Immunofluorescent signals in muscle fibres were also quantified in GM muscle. In this muscle, COXIV signal intensities did not differ as a function of $\alpha / \beta$ CaMKII overexpression (Figure 5D), but a larger fraction of CaMKII-transfected than non-transfected muscle fibres demonstrated MHCII staining (0.77 vs. $0.12, \mathrm{p}=0.048$; Chi2-Test).

\section{CaMKII signalling after paced contractions in situ}

We investigated whether $\alpha / \beta$ CaMKII-transfected SOL muscle retains responsiveness for contraction-induced CaMKII signalling. Paced isometric exercise in situ increased threonine-287 phosphorylation of $\beta$ CaMKII (Figure 6A). Concomitantly, muscle fibres with elevated CaMKII content demonstrated increased signal for pThr287-CaMKII (Figure 6B-E). RT-PCR experiments demonstrated reduced transcript levels of mitochondrial factors (COXI, COXIV, SDHB) and SERCA2A immediately after high-intensity exercise (Figure $5 \mathrm{~B}$ ).

\section{Transfection affects muscle contractility}

Compared to non-transfected muscle, twitch force $(-12 \%)$ and maximal tetanic force $(-30 \%)$ were reduced and time-to-peak-twitch force $(+22 \%)$ and half-relaxationtime $(+32 \%)$ were prolonged in control-transfected muscle (Table 1). When values were assessed separately for the

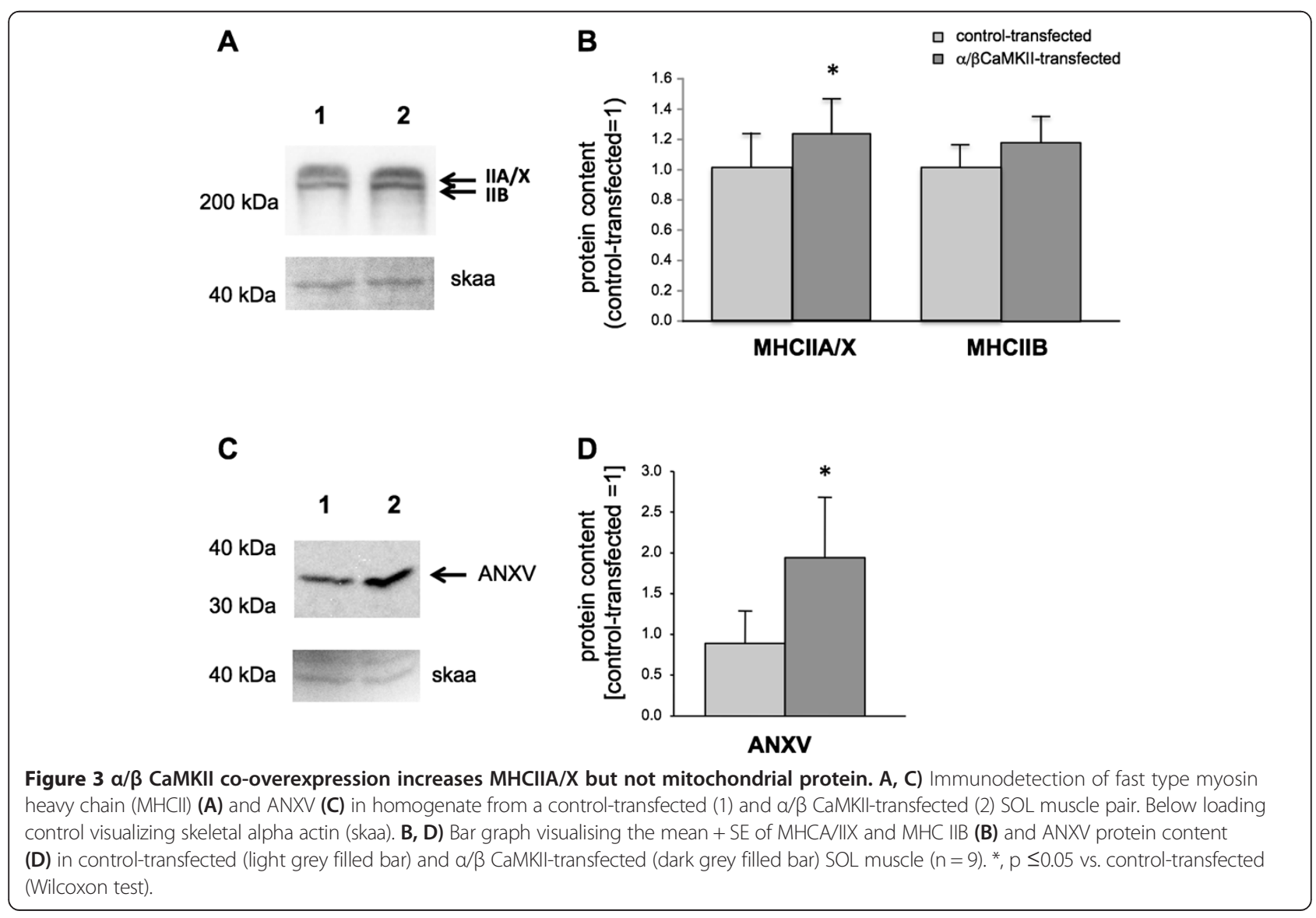


A

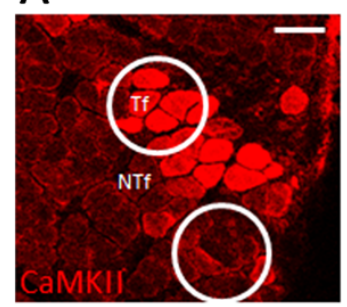

D

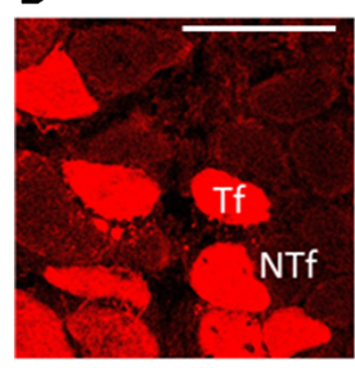

B

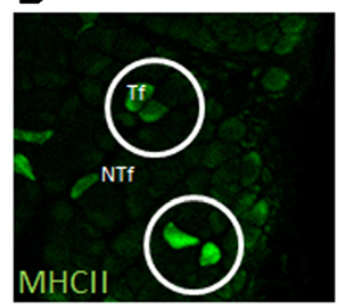

$\mathrm{E}$

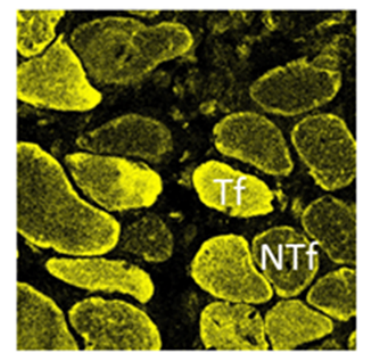

C

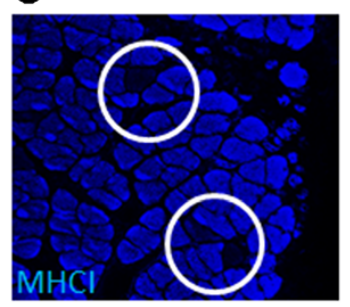

$F$

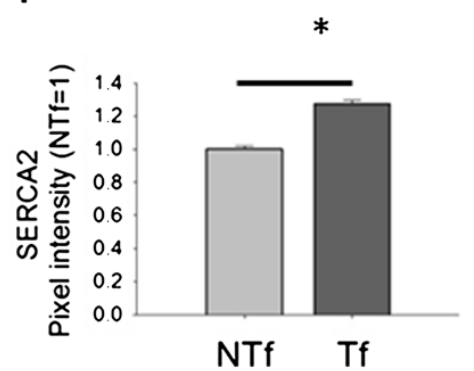

Figure $4 \alpha / \beta$ CaMKII co-overexpression in relation to MHC and SERCA2 expression. A-C) Microscopic field of a/ $\beta$ CaMKII-transfected SOL muscle stained for CaMKII (A, red), fast MyHCII (B, green) and slow $\mathrm{MHCl}(\mathbf{C}$, blue). Corresponding areas are circled. $\mathbf{D}$, E) Microscopic field of an a/B CaMKII-transfected SOL muscle after staining for CaMKII (red, D) and SERCA2 (yellow, E). F) Bar graph visualizing the mean + SE of SERCA2 levels in transfected $(T f, n=276)$ and non-transfected ( $N T f, n=221)$ muscle fibers. ${ }^{*}, p \leq 0.001$.

two muscles, twitch force and maximum tetanic force were reduced in SOL muscle with control-transfection. Concomitantly, markers of damage (caveolin 3 mRNA) and regeneration (myogenin protein, fibres with internal nuclei) were elevated in SOL muscle 7 days after transfection (Figure 7).

\section{Discussion}

This is the first study that investigates whether increased CaMKII protein expression affects the skeletal muscle phenotype in vivo. Transcript measures indicate that $\alpha / \beta$ CaMKII signalling down-regulates mitochondrial gene expression after high-intensity exercise (Figure 5B). In contrast to our hypothesis, we did not observe altered expression of mitochondrial protein(s) in $\alpha / \beta$ CaMKII-transfected muscle fibres of rats housed under normal cage activity (Figure 5A/D). By contrast, twitch contraction and relaxation times were reduced in $\alpha / \beta$ CaMKII-transfected muscles (Figure 2) when major proteins of muscle contraction and relaxation, i.e. $\mathrm{MHC}$ IIA/IIX and SERCA2, were increased (Figures 3A/B and 4). Regarding contractile features and MHCII expression in transfected muscle (fibres) the effects of $\alpha / \beta$ CaMKII overexpression were comparable in the slow-twitch and fast-twitch muscle under investigation. Our results suggest that, in the context of muscle transfection, $\alpha / \beta$ CaMKII content regulates the expression of proteins involved in muscle contraction and relaxation, but not of proteins involved in mitochondrial biogenesis.

\section{Technical considerations}

In order to assess the effect of an increase in CaMKII holoenzymes, we deployed gene electro-transfer with expression constructs for native $\alpha$ - and $\beta$-CaMKII isoforms with similar substrate specificity and structure as the skeletal muscle CaMKII isoforms [35,36]. CaMKII overexpression was confined to muscle fibres (Figures $4 \mathrm{~A}, 5 \mathrm{C}$ and 6B/D), in which CaMKII content was increased 4.0-5.3 fold compared to CaMKII content in non-transfected fibres. The latter possibly reflects the abundant $\beta \mathrm{M}$ isoform (Figure 1A; [36,41]). Immunodetection of the validated protein band for threonine 287-phosphorylated CaMKII (Figure 1C) demonstrated that the introduced $\beta$ CaMKII retained responsiveness for contraction-induced CaMKII signalling (Figure 6A). The findings imply that the increased content of native CaMKII resulted in potentially higher CaMKII activity which is amenable to physiological regulation during muscle recruitment in vivo. It has been estimated that motor units in SOL muscles of caged, but freely moving rats are active for $25-30 \%$ during a 24-hour period [34]. Furthermore, rat SOL is recruited during postural and slow running activity [37]. Therefore, we expected transfected fibres in this muscle to be frequently recruited. We acknowledge, however, that muscle regeneration is inherent to the selected mode of transfection (Figure 7). This is of relevance for our finding that mitochondrial protein levels in $\alpha / \beta$ CaMKII-transfected SOL muscles were unaffected because muscle regeneration decreases mitochondrial enzyme expression and activity $[42,43]$. 

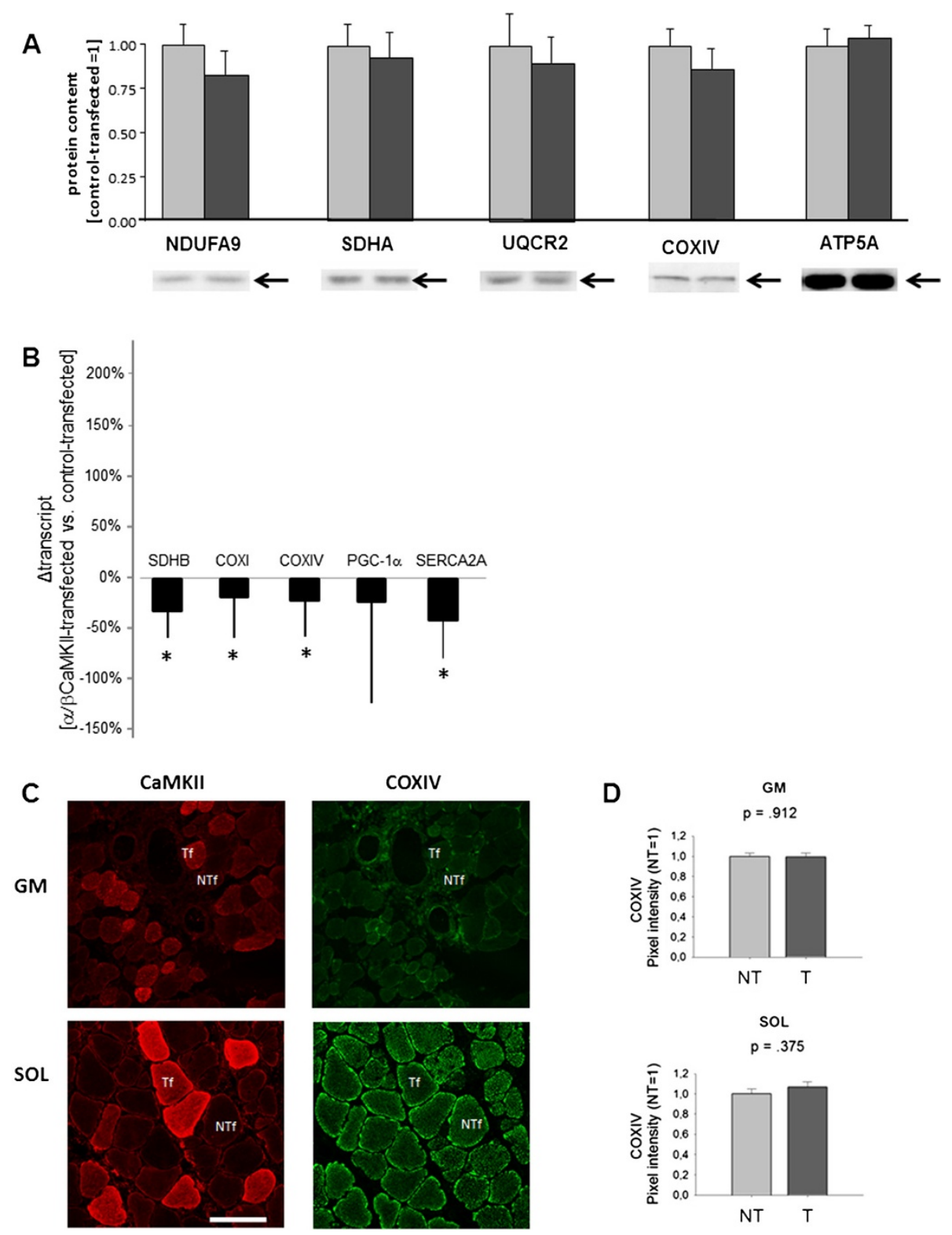

Figure 5 Effect of $\alpha / \beta$ CaMKII co-overexpression on mitochondrial gene expression. A) Bar graph visualising the mean + SE of assessed mitochondrial proteins in control-transfected (light grey filled bar) and a/ $\beta$ CaMKII-transfected (dark grey filled bar) SOL muscle $(n=6)$. Representative examples of detected mitochondrial protein are shown below. *, $p \leq 0.05$ vs. control-transfected (Wilcoxon Test). B) Bar graph of mean + SE of the differences in expression of selected gene transcripts between a/ $\beta$ CaMKIl-transfected and control-transfected SOL muscle $(n=6)$. ${ }^{*}$ denotes $p \leq 0.05$ vs. control-transfected muscle (T-test). C) Microscopic images visualising the association of the mitochondrial marker, COXIV, with transfected (Tf) and non-transfected (NTf) fibres in a/ $\beta$ CaMKII-transfected GM and SOL muscle. D) Bar graph showing the mean + SE of COXIV levels in transfected ( $n=54$ for $\mathrm{GM}$ and $\mathrm{n}=38$ for SOL) and non-transfected ( $\mathrm{n}=51$ for $\mathrm{GM}$ and $\mathrm{n}=35$ for SOL) muscle fibres. The corresponding $p$-values are indicated.

The identified reduction in time-to-peak-twitch force and half-relaxation time in $\alpha / \beta$ CaMKII-transfected muscles (Figure 2) support a role of CaMKII content in the regulation of excitation-contraction coupling in skeletal muscle. The comparison to non-transfected muscle reveals that this contractile effect occurred on the background of reduced contractility with transfection-induced muscle damage and regeneration (Table 1; Figure 7). Considering the broad influence of muscle damage on myogenic processes and the expression of factors involved in excitation-contraction coupling $[44,45]$; a number of factors are likely involved in correcting the detriment in contractility by the overexpression of $\alpha / \beta$ CaMKII. This potentially includes the calcium channels, SERCA2 and RyR, which control excitation-contraction coupling via calcium release and re-uptake in the sarcoplasmic 
A

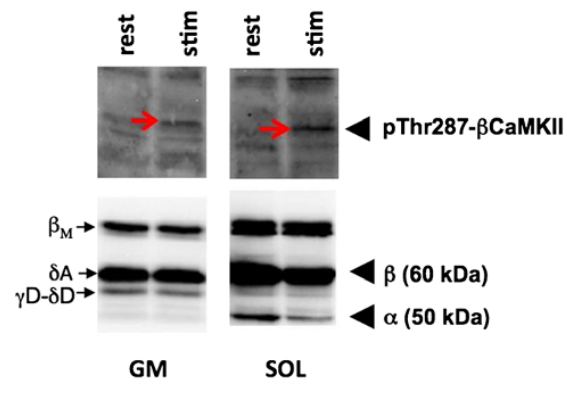

B

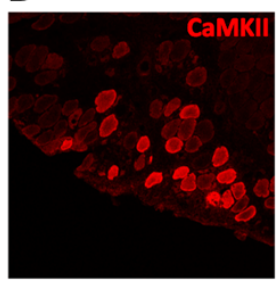

D

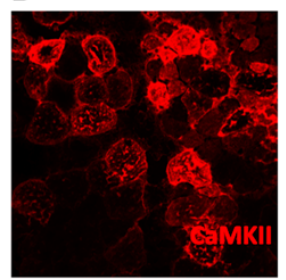

C

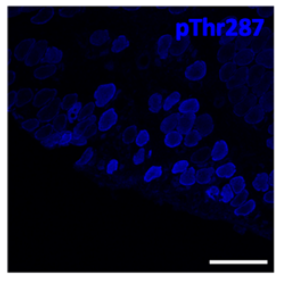

E

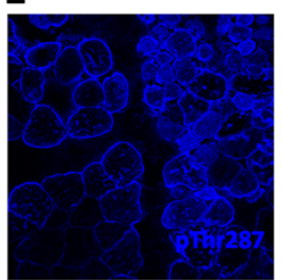

Figure 6 Phosphorylation of overexpressed CaMKII after isometric exercise in situ. A) Example of immunodetected CaMKII (top panel) and pThr287-CaMKII (bottom) in transfected GM and SOL muscle after 2-minutes of isometric exercise in situ. Endogenous and exogenous (bold font) CaMKII isoforms are labelled. Red arrows indicate threonine 287 phosphorylated $\beta$-CaMKII in lanes loaded with homogenate from stimulated samples (stim) compared to non-stimulated samples (rest). B, C) Microscopic field of a/ $\beta$ CaMKII-transfected SOL muscle after staining for CaMKII (red, B) and pThr287-CaMKII (blue, C). D, E) Microscopic field of a/ $\beta$ CaMKII-transfected GM muscle after staining for CaMKII (red, D) and pThr287-CaMKII (E). Note the congruency of CaMKII and pThr287-CaMKIl stained muscle fibres. Scale bar denotes $250 \mu \mathrm{m}$.

reticulum. In vitro and in isolated muscle fibres both channels are regulated by CaMKII-dependent phosphorylation ([22,46], Hawkins et al., [47,48]). The characterisation of CaMKII-mediated phosphorylation was not the focus of this investigation. At present it is therefore unclear if CaMKII-dependent phosphorylation of SERCA and RyR played a role in decreasing twitch contraction and relaxation times in our experiments. Rather we identify that correction of the detriment in time-to-peak contraction and half-relaxation time by $\alpha / \beta$ CaMKII overexpression was associated with altered expression of SERCA2 and selected factors involved in excitationcontraction coupling (i.e. MHCIIA/X, ANXV). The increased SERCA2 protein levels in SOL were localised to muscle fibres that overexpress $\alpha / \beta$ CaMKII (Figure 4D-E). The localisation of SERCA2 and ANXV to the sarcoplasmic reticulum $[49,50]$ suggests that the decrease in half-relaxation time with $\alpha / \beta$ CaMKII overexpression involves adjustments within the sarcoplasmic reticulum of transfected muscle fibres.

By contrast we observed no effect of $\alpha / \beta$ CaMKIItransfection on maximal force production (Figure 2). Regeneration has been shown to decrease specific force and increase the ratio of twitch force to tetanic force [44]. This detriment has been shown to last up to seven days after injury [43]. Therefore, it may be that any increase in maximal force was masked by negative effects of transfection on characteristics that limit force production with contraction.

Transfection of SOL muscle is associated with the expression of myosin heavy chain IIA (MHCIIA) in type I muscle fibres [39]. In this regard it is of interest that $\alpha / \beta$ CaMKII overexpression in slow-twitch $\mathrm{SOL}$ muscle further increased MHCIIA/X levels compared to control-transfected muscle (Figure $3 \mathrm{~A}$ ) and that the CaMKII-transfected muscle fibres demonstrated

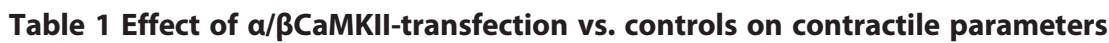

\begin{tabular}{|c|c|c|c|c|c|c|c|c|}
\hline \multirow[b]{3}{*}{$F_{\text {twitch }}(\mathrm{mN})$} & \multicolumn{4}{|l|}{ SOL } & \multicolumn{4}{|l|}{ GM } \\
\hline & \multirow{2}{*}{$\frac{\text { Non-transfected }}{2169.3}$} & \multicolumn{2}{|c|}{ Control-transfected } & $\alpha / \beta C a M K I I-t r a n s f e c t e d$ & Non-transfected & \multicolumn{2}{|c|}{ d Control-transfected } & \multirow{2}{*}{$\begin{array}{l}\alpha / \beta C a M K I I-t r a n s f e c t e c \\
2380178\end{array}$} \\
\hline & & 17815 & $\mathrm{a}$ & 17420 & 254886 & 2377175 & a & \\
\hline $\mathrm{F}_{\max }(\mathrm{mN})$ & 108357 & 55557 & a & 56880 & 8864395 & 7594638 & a & 8078723 \\
\hline Fatigue (\%) & 10.53 .6 & 18.85 .3 & & 22.75 .4 & 67.52 .1 & 5.32 .9 & 67.62 & \\
\hline TTP (ms) & 10514 & 1337.9 & a & $1087.3^{b}$ & 43.03 .3 & 50.94 .0 & a & $44.22 .4^{b}$ \\
\hline HRT (ms) & 12118 & 1684.6 & a & $1426.9^{b}$ & 42.95 .9 & 54.06 .0 & a & $46.23 .9^{b}$ \\
\hline
\end{tabular}

Data are shown as mean ? SE. For abbreviations see Figure 2. Fatigue: \% decrease in force after the 50 maximal tetanic contractions of the high-intensity exercise, relative to first contraction; ${ }^{a}$ : Main effect of electro-transfer procedure $(p<0.05){ }^{\text {b }}$ : Main effect of $\alpha / \beta C a M K I I$ overexpression $(p<0.05)$. 

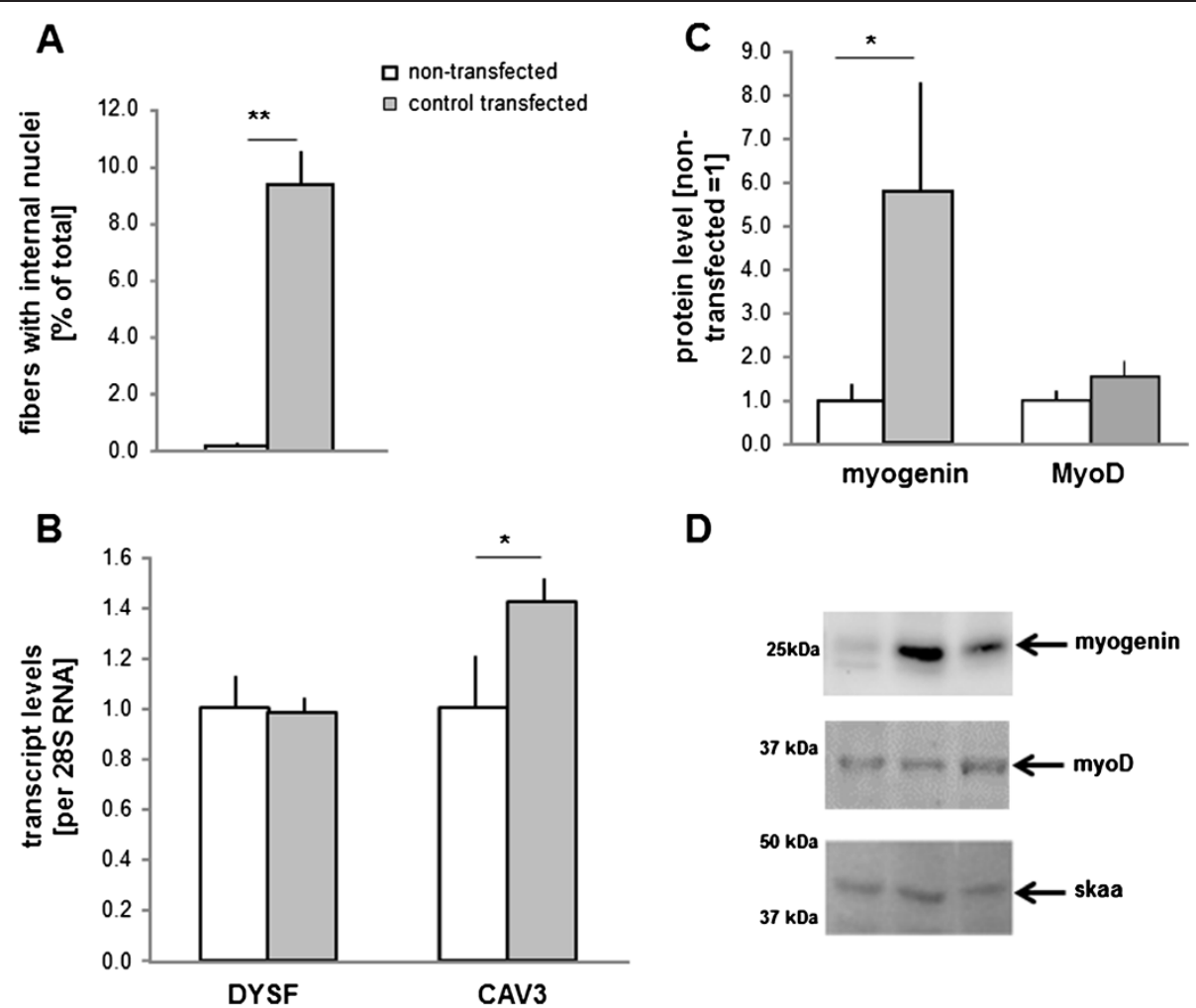

D

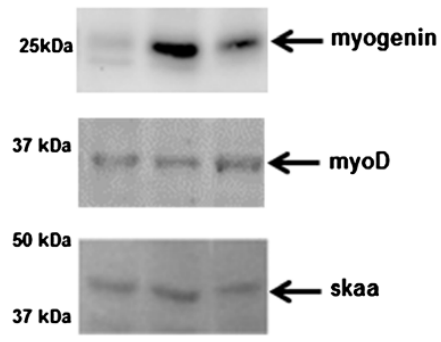

Figure 7 Damage and regeneration with electroporation. A, B) Bar graph showing mean + SE of the percentage of fibers with central nuclei (A) and levels of the damage markers, caveolin 3 and dysferlin mRNA, in non-transfected and control-transfected SOL muscle (B). C, D) Bar graph of the mean + SE (C) and a representative immunodetection (D) of the myogenic marker myogenin (top), myoD (middle) and the skaa loading control (bottom) in (from left to right) non-transfected, control-transfected and a/b-transfected muscle ( $n=8$, each). Proteins of interest are indicated with an arrow respective to the position of molecular weight markers (in $\mathrm{kDa}$ ). ${ }^{*}$ and ${ }^{* *}$, p-values $<0.05$ and $<0.001$ for the indicated differences (unpaired T-test).

an increased fraction of MHCII expression in SOL (0.36 vs. 0.18$)$ and $\mathrm{GM}$ muscle $(0.77$ vs. 0.12$)$. The identified increase in SERCA2 and ANXV content with $\alpha /$ $\beta$ CaMKII-overexpression compare to the demonstrated influence of overexpressed native CaMKII on gene expression being associated with excitation-contraction coupling and hypertrophy in cardiac myocytes [51,52]. Our findings are also in accordance with the results of Allen \& Leinwand [19], who demonstrated that the calcium-ionophore A23187 increased MHCIIA promoter activity in $\mathrm{C} 2 \mathrm{C} 12$ cells, which was attenuated by the CaMK inhibitor KN62. This raises the hypothesis that an increase in sarcoplasmic calcium levels in injured muscle fibres enhances expression of the fast fibre type program, through a CaMKII-dependent mechanism.

In contrast to our expectations, we observed no increase in mitochondrial protein (Figure $5 \mathrm{~A}$ ) and fatigue resistance (Figure $2 \mathrm{~B}$ ) in $\alpha / \beta$ CaMKII-overexpressing muscles compared to control-transfected muscles. To the best of our knowledge, this is the first study investigating whether increased content of native CaMKII increases the expression of a mitochondrial protein in any tissue type. Constitutively active CaMKIV increases mitochondrial biogenesis when overexpressed from an embryonic stage onward [18], but this CaMK is not endogenously expressed in skeletal muscle $[23,32]$ and CaMKIV knock-out mice do not display altered muscle adaptation in response to training [23]. Whether CaMKIV would have a similar function in skeletal muscle compared to CaMKII is questionable, since the two proteins have different substrate specificity and intracellular localisation [53-55].

The results demonstrate that increased $\alpha / \beta$ CaMKII content is not sufficient to increase mitochondrial gene expression. We can therefore not rule out the possibility that CaMKII is required in conjunction with other signalling pathways for the response to exercise as shown before for the activity of a GLUT4-enhancer in mouse $m$. tibialis anterior [56]. In this regard, we identify that the reduced mitochondrial and SERCA2 transcript levels after high-intensity exercise in $\alpha / \beta$ CaMKII-transfected SOL muscle (Figure 5B) reproduce the reduced transcript expression within hour after high-load type of bicycle exercise [57] which contrasts to the up-regulation of gene transcripts with low-load endurance type exercise [58]. This suggests that effects of regeneration on 
mitochondrial transcript expression possibly interact with insufficient endurance type stimuli in the $\alpha / \beta$ CaMKII-transfected muscle of the rats housed under normal cage activity.

\section{Conclusion}

Our results support a role for elevated sarcoplasmic CaMKII content for accelerating muscle contraction and relaxation in regenerating muscle via effects that include the enhanced expression of fast myosin heavy chain and sarcoplasmic reticulum associated SERCA2 and ANXV protein, but not in mitochondrial biogenesis. These observations in vivo are the first to point out a role for quantitative changes in this multi-functional calcium-dependent enzyme in control of the contractile muscle phenotype.

\section{Methods}

\section{Experimental design}

Three experimental groups from two phenotypically distinct muscles (i.e. the fast GM and slow SOL muscle) were compared in this experiment: group 1: $\alpha / \beta$ CaMKII-transfection, group 2: control-transfection, and group 3: non-transfected controls. The comparison of group 1 vs. 2 allows identifying the feasibility and effect of CaMKII overexpression, while the comparison of groups 2 vs. 3. allows conclusions on the effects of transfection alone.

Transfection of muscle fibres was achieved via intramuscular injection of plasmid prior to the application of defined electric pulses (electro-transfer). Thereby a paired design was adopted where $\alpha / \beta$ CaMKII-transfected right SOL and GM muscles of animals were compared to the respective contralateral (i.e. left) muscles that were subjected to a control-transfection.

Effects were assessed seven days after the intervention through measurements of selected molecular, cellular and functional parameters. Functional effects were assessed in situ in intact muscle-tendon preparations (for abbreviations see Figure 2). The molecular measures included the quantification of the content in selected proteins using western blotting/immunodetection and the content of selected gene transcripts after high-intensity exercise in situ. The specificity of detecting CaMKII and threonine 287 phosphorylated CaMKII (pThr287-CaMKII) was assessed based on western blotting and immunodetection of homogenates from control-transfected and $\alpha / \beta$ CaMKIItransfected muscle, and homogenates which were incubated in vitro to assess calcium/calmodulin-dependent threonine 287 phosphorylation with commercial antibodies. The cellular specificity of CaMKII overexpression and downstream effects were assessed by quantifying immunofluorescent signals as recorded using confocal microscopy or by quantifying conventional colorimetric staining as documented with light microscopy.

\section{Animals}

Female Wistar rats were used for the experiments described here. In situ contraction protocols and the majority of the electroporation experiments were carried out at the MOVE Research Institute Amsterdam, VU University Amsterdam, The Netherlands, and approved by the local committee on ethics of animal experimentation. Rat handling and experiments conformed with the Dutch Research Councils guide for the care and use of laboratory animals. Two series of electroporation experiments were carried out at the Department of Cardiovascular Surgery, University Hospital Bern, Switzerland. These experiments were carried out according to the permission of the Animal Care Committee of the Canton of Berne (Switzerland) and following the recommendations provided by the European Convention for the protection of Vertebrate Animals used for Experimental and Scientific purposes (Strasbourg, 18.III.1986).

\section{Transfection}

Plasmid injection followed by electropulsing was essentially carried out with modifications as described [39]. Three-month-old female Wistar rats (Harlan Laboratories/ Charles River; 191-230 grams, $n=13$ ) were used to transfect GM and SOL muscle. Both, left and right muscles were injected with a reporter plasmid prior to electropulsing; the right muscles only were also injected with plasmid for native $\alpha$ and $\beta$ CaMKII. pCDNA3 plasmids vectors encoding full-length cDNA for $\alpha$ CaMKII (pCDNA3-CaMKII $\alpha$ ) and $\beta$ CaMKII (pCDNA3-CaMKII $\beta$ ) were a gift from Dr. M Neal Waxham (University of Texas, Houston, USA). The reporter plasmid encoding full-length luciferase under control of 424 basepairs upstream of the transcription start site of the chicken skeletal $\alpha$-actin gene [59] was a gift from Dr. Frank W. Booth (University of Missouri, Columbia, USA). Animals were anaesthetized with $2-4 \%$ isoflurane through inhalation. Hindlimbs were shaved, and skin was disinfected with $70 \%$ ethanol. An incision was made into the skin and fascia between GM and $m$. tibialis anterior. SOL muscle was subsequently exposed and liberated, after which four injections of a plasmid mixture with a total volume of $90 \mu \mathrm{l}$ were administered intramuscularly with a 29-gauge insulin syringe. Subsequently four injections of a total volume of $180 \mu \mathrm{l}$ were administered to the GM along the length of the muscle. 5 minutes after DNA injection, the muscles were subjected to electroporation as established. 6-mm long needle electrodes (BD microlance TM3, 27G 12"; distance $4 \mathrm{~mm}$ ) were inserted perpendicular to the fibre orientation of the injected muscle and subjected to discrete pulse protocols as established in previous experiments. For SOL the protocol involved the delivery of 
3 trains of 80100 microsecond pulses at $100 \mathrm{~mA}$, with 992 milliseconds of interrupt on 2 locations using needle electrodes with a GET42EV generator (E.I.P. Electronique et Informatique du Pilat, Jonzieux, France) [39]. For the GM, this included the delivery of 8 trains of 60100 microsecond duration at $50 \mathrm{~mA}$, with 994 milliseconds interrupt on 3 locations. A mix of expression plasmid pCDNA3-CaMKII $\alpha\left(0.22 \mu \mathrm{g} \mu \mathrm{l}^{-1}\right)$ and pCDNA3CaMKII $\beta\left(0.22 \mu \mathrm{g} \mu \mathrm{l}^{-1}\right)$ in TBE buffer was injected into muscles of the right leg together with the reporter plasmid $\left(0.55 \mu \mathrm{g} \mu^{-1}\right)$. Data obtained from this reporter construct are beyond the scope of this paper, and are therefore not reported. Muscles of the left leg were injected with the reporter plasmid only $\left(1 \mu \mathrm{g} \mu \mathrm{l}^{-1}\right)$. Right and left muscles of this experiment will henceforth be referred to as $\alpha / \beta$ CaMKII-transfected and control-transfected, respectively. For the study of CaMKII activation by isometric exercise in situ, both left and right muscles were transfected with $\alpha / \beta$ CaMKII plasmid.

After electropulsing, the skin wound was closed with sutures, and the animal was allowed to recover from anaesthesia. Animals were kept in cages afterwards, where they resumed normal activity within hours after surgery. After seven days, animals were anaesthetized for further measurement of contraction parameters (see below) and euthanized by intra-cardiac injection of Euthasol (VU University Amsterdam) or anaesthetized with $3 \%$ isoflurane and euthanized by dislocation of the cervical vertebrae and rapid exsanguination (University Hospital Bern). Treated muscles were harvested from both legs and snap-frozen in liquid nitrogen.

\section{In situ measures of muscle contraction}

For measurement of isometric muscle contraction parameters, rats were anaesthetized by intra-peritoneal injections of $1.2 \mathrm{ml} / 100$ gram body weight of $12.5 \%$ urethane [60]. Ear and foot reflexes were tested to check whether the animal was sufficiently anesthetized. Subsequent injections of $0.3-0.5 \mathrm{ml}$, up to a maximum of $1.5 \mathrm{ml}$, were given every $10 \mathrm{~min}$ afterwards until reflexes had disappeared. Experiments were carried out at room temperature $(24 \mathrm{C})$. Rats were kept on a heated pad to prevent hypothermia. Hindlimbs were shaved and skin was removed, after which GM and SOL muscles were exposed and mechanically isolated by removing as much as possible the myofascial connections to surrounding muscles. Blood supply to, and nerve innervations of, both muscles were kept intact and tendons of GM and SOL muscles were attached to a force transducer via Kevlar wires. The sciatic nerve was severed proximally and connected to an external electrode that was controlled by a computer to receive pulse wave stimulation.

Optimum length of the muscle-tendon complex (the length of the muscle-tendon complex at which maximum tetanic force was produced) for isometric contractions was first estimated using twitches, then determined using a protocol consisting of two twitches and one tetanic contraction (pulse duration $100 \mu \mathrm{sec}$, tetanic stimulation frequency: $100 \mathrm{~Hz}$, train duration: $400 \mathrm{~ms}$ [61]. Muscles were kept below slack length between contractions and rest duration between maximal contractions was approximately one minute. After determination of optimum length, muscles rested for five minutes. Subsequently, while muscles were set to optimum length, a protocol of high-intensity exercise (stimulation frequency: $100 \mathrm{~Hz}$; train duration: $300 \mathrm{~ms}$, one train every $800 \mathrm{~ms}, 50$ trains/contractions) was applied to induce muscle fatigue. GM and SOL muscles were dissected after the end of stimulation and snap-frozen in liquid nitrogen. Animals were killed by intra-cardiac injection of Euthasol, while still fully anaesthetized. Frozen muscles were stored at $-80 \mathrm{C}$ until use for western blotting/immunodetection and microscopic analysis as described below.

Force data during muscle stimulation were sampled at a frequency of $1000 \mathrm{~Hz}$ and collected using custom written software based on Matlab (v 7.5.0, The Mathworks Inc., MA, USA). Time-to-peak-twitch force, half-relaxation time, maximum twitch force and maximum tetanic force were determined. The same contraction parameters were determined for a group of non-transfected muscles (NT; SOL/GM, $n=8$ ). Values obtained for the two twitch values in each trace were averaged.

\section{Isometric exercise in situ}

A two-minute stimulation protocol consisting of intermittent isometric tetanic contractions of GM muscle at $100 \mathrm{~Hz}$ stimulation frequency [27] was applied via the sciatic nerve to $\alpha / \beta$-CaMKII-transfected SOL muscle in situ. The stimulated muscles $(n=6)$ were freeze-clamped between liquid nitrogen-cooled aluminium grips during stimulation after two minutes. Non-stimulated, $\alpha / \beta$-CaMKII-transfected contra-lateral muscles were subsequently dissected and frozen in liquid nitrogen. Proteins were extracted from the muscle and subjected to SDS-PAGE followed by western blotting and immunodetection as described below.

\section{Protein biochemistry \\ Western blotting/immunodetection}

To analyse protein expression, frozen $25 \mu \mathrm{m}$ thick cross-sections taken from the centre portion of the muscle were homogenized in ice-cold RIPA buffer (50 mM TRIS-HCl (pH 7.5), $150 \mathrm{mM} \mathrm{NaCl}, 1 \mathrm{mM}$ EDTA, $1 \% \mathrm{v} / \mathrm{v}$ Nonidet P40 substitute, $0.25 \% \mathrm{w} / \mathrm{v}$ sodium deoxycholate) plus freshly added protease/phosphatase inhibitors: $1 \mathrm{mM} \mathrm{NaF}, 1 \mathrm{mM} \mathrm{Na} \mathrm{VO}_{4}, 0.1 \mathrm{mM}$ PMSF, $1 \mu \mathrm{g} \mathrm{m}{ }^{-1}$ leupeptin, $0.2 \mu \mathrm{g} \mathrm{ml}^{-1}$ pepstatin, $0.1 \mu \mathrm{g} \mathrm{ml}^{-1}$ aprotinin, using a Polytron homogenizer (Kinematica, 
Luzern, Switzerland). Chemicals were obtained from Sigma-Aldrich (Dorset, United Kingdom). Crude homogenates were aspirated 5-10 times through a $0.8 \mathrm{~mm}$ syringe needle, and stored at $-80 \mathrm{C}$ until use for analysis. An aliquot of the aspirated homogenate was taken for determination of protein concentration with the bicinchoninic acid protein assay (Pierce, Rockford IL, USA).

Protein levels of total CaMKII and pThr287-CaMKII were analysed by western blotting followed by immunodetection. Homogenates were denatured by addition of SDS-PAGE buffer (final concentration: $50 \mathrm{mM}$ TRIS- $\mathrm{HCl}$ (pH 6.8), $2 \% \mathrm{w} / \mathrm{v}$ bromophenol blue, $10 \% \mathrm{v} / \mathrm{v}$ glycerol, $2 \% \beta$ mercaptoethanol) and five minutes heating at $95 \mathrm{C}$. 20-40 $\mu$ g of protein was separated by SDS-PAGE and transferred overnight onto a nitrocellulose membrane (GE Healthcare, Little Chalfont, UK). Membranes were stained with Ponceau $\mathrm{S}$ solution to confirm equal protein loading and transfer. The membrane was blocked in 5\% skimmed milk in TRIS-buffered saline $(\mathrm{pH}$ 7.4) with 0.05\% Tween-20 (TBS-T), followed by incubation with a primary antibody for pan-CaMKII (BD Bioscience \#611292, dilution: 1/2500), pThr287-CaMKII (Cell Signalling Technology \#3361, dilution: 1/1000), fast myosin heavy chain (Sigma-Aldrich \#M4276, dilution 1/1000), myogenin (Santa Cruz Biotechnology, sc-12732 (F5D); 1:200) or myoD (Santa Cruz Biotechnology, sc-304; 1:200) or OxPhos proteins (succinate dehydrogenase Fp subunit (SDHA), ATP synthase subunit $\alpha$ (ATP5A), ubiquinol cytochrome $\mathrm{c}$ oxioreductase subunit 2 (UQCRC2) and NADH ubiquinone oxidoreductase subunit 9 (NDUFA9); \#458199, Invitrogen, dilution $1 / 1000$, and COXIV (\#4850, Cell Signalling Technology, 1/2000)) for 2 hours. Antibody incubation solutions were 5\% milk or $5 \%$ bovine serum albumin (BSA) in TBS-T. Finally, membranes were incubated with species-specific horseradish peroxidase-conjugated secondary antibodies (Millipore, Watford, UK). Membranes were washed in TBS-T for 45 minutes after both antibody incubations. Antibodies were detected with an enhanced chemiluminescence kit (Pierce, Rockford IL, USA). Light signals were captured with a ChemiDoc XRS system (Biorad, Hemel Hempstead, UK).

Transfected muscle pairs from the same animal were run on the same blot. Measures were limited to animals whose CaMKII injected \& porated muscles showed increased expression in either of the exogenous CaMKII isoforms on a western blot. Protein bands were quantified with Quantity One version 4.6.8 (Biorad). These values were subsequently expressed as relative to the mean of the control-transfected muscles for the respective immunoblot.

\section{Establishing CaMKII detection}

To identify CaMKII isoforms in skeletal muscle [41] buffer components were added to muscle homogenate to promote, or suppress, calcium/calmodulin dependent protein phosphorylation in vitro as described [62]. The reaction products were subjected to SDS-PAGE and immunoblotted for CaMKII as described in the section on western blotting and immunodetection below. CaMKII isoforms were labelled in reference to a previous report [41]. The phosphorylated form of exogenous $\beta$ CaMKII was identified based on its molecular weight $(60.4 \mathrm{kDa})$, which is very similar to that of $\delta a(60.1 \mathrm{kDa})$, the second largest CaMKII isoform in skeletal muscle [29].

\section{Microscopic analysis}

To investigate differences in protein expression at the single fibre level, immunofluorescent staining was performed on cryosections of transfected muscles. Sections $(12 \mu \mathrm{m}$ thickness) were cut on a cryostat (CM3050, Leica, Germany) and dried for 30 minutes on glass slides. Sections were then fixed with ice-cold acetone and blocked with 5\% normal goat serum in phosphate buffered saline, pH 7.5 (PBS). CaMKII and SERCA2, COXIV, MHCI or MHCII, were detected simultaneously using commercially available primary antibodies (anti-CaMKII; anti-SERCA2 (Abcam \#Ab3625 or Abcam \#2A7-A1, dilution 1/200); antiCOXIV (\#4850, Cell Signalling Technology); anti-MHCI (\#MAB1628, Millipore, dilution 1/100); anti-MHCII (\#M4277, Sigma Chemicals, dilution 1/100) and speciesspecific Alexa 488/555 secondary antibodies (Invitrogen). Sections were washed with PBS for 45 minutes after both antibody incubations. To detect nuclei, sections were incubated for 10 minutes with TO-PRO-3 iodide (Invitrogen, Paisley, UK). Immuno-labelled sections were embedded in fluorescence compatible mounting medium (DAKO, Ely, UK).

Protein expression in electroporated fibres was analysed on a TCS SP5 confocal microscope (Leica, Milton Keynes, UK). A 10 objective was used in combination with 4 optical zoom. The fluorescent labels were excited with an Argon laser at $488 \mathrm{~nm}$ and $\mathrm{HeNe}$ lasers at $543 \mathrm{~nm}$ and $633 \mathrm{~nm}$. The pinhole was set to match the thickness of the stained section and the focus plane was adjusted to maximize signal detection. Dyes were excited separately using a sequential scanning mode. Detected light spectra were set to maximize signal detection, but care was taken to prevent crossexcitation of dyes. Laser intensity was set to produce images with few under- or overexposed pixels, and low levels of non-specific staining, as indicated by light emission from sections that had been labelled with the secondary antibodies only. 8-Bit images were captured at 20482048 pixels, using $100 \mathrm{~Hz}$ scanning speed and 5-times line averaging.

The efficiency of overexpression was estimated by taking a tile scan of one cryosections of the belly portion of each $\alpha / \beta$ CaMKII-transfected muscle, which was stained 
for CaMKII using immunofluorescence or colorimetric staining. Subsequently individual microscopic fields were assessed for the number of fibres with prominent staining for CaMKII and the total fiber number. On average 234 and 386 fibres were counted for SOL and GM muscle, respectively. MCSA of transfected and non-transfected fibres was estimated in colorimetrically stained cross sections using Adobe Photoshop CC (Adobe Systems Incorporated) in regions of $\alpha / \beta$ CaMKII-transfected SOL muscle as described [63]. Only fibres which met the criteria of a circularity factor $>0.5$ were included. On average 30 transfected and 60 non-transfected fibres from the same transfected region were assessed per cross section.

SERCA2, COXIV and CaMKII staining intensity in muscle fibres overexpressing CaMKII (identified based on CaMKII staining intensity by visual inspection) was quantified with ImageJ (rsbweb.nih.gov/ij/). Fibres were circumscribed manually and the average pixel intensity within the fibre was measured. An approximately equal number of fibres in the same image which did not demonstrate elevated CaMKII content (designated non-transfected fibers) was measured as well, and acted as the control group of fibres to which the CaMKII-overexpressing fibres (designated transfected fibers ) were compared. MHCII expression in transfected and non-transfected fibers was assessed in the transfected region based on the presence or absence of MHC-staining as this staining was rather discrete than continuous.

Fibres with internal nuclei were assessed on $12-\mu \mathrm{m}$ cryosections being stained with and counterstained with hematoxylin (MERCK, Germany). Microscopic files were taken at a 10-fold magnification (Axioskop 2, Carl Zeiss Ltd, Welwyn Garden City, UK) and assembled with AxioVision software (Carl Zeiss Ltd). Subsequently a grid with $250-\mu \mathrm{m}$ unit length was superimposed and microscopic fields corresponding to $0.0625 \mu \mathrm{m} 2$ were assessed in a random initiation, systematic manner counting fibre profiles and fibre profiles with internal (blue) nuclei. On average $475+50$ fibre were counted per muscle.

\section{RT-PCR}

RNA extraction from muscle tissue and RT-PCR analysis were carried out as described elsewhere [64]. Total RNA was extracted from frozen $25 \mu \mathrm{m}$-sections of transfected SOL muscles using the RiboPure kit (Applied Biosystems). RNA concentration and purity (260/280 nm ratio; mean: 2.06, range: $1.92-2.09$ ) were determined using a spectrophotometer (Nanodrop Technologies, Wilmington, DE). Total RNA concentration in muscle tissue was expressed as RNA (ng) per weight of the analysed sample (mg). Five hundred nanogram of total RNA per muscle was reversetranscribed using the high capacity RNA-to-cDNA kit (Applied Biosystems) containing random primers in a $20 \mu \mathrm{l}$ total reaction volume. Tubes were heated at $25 \mathrm{C}$ for
$5 \mathrm{~min}$, followed by $42 \mathrm{C}$ for $30 \mathrm{~min}$. Finally; the tubes were heated to $85 \mathrm{C}$ for $5 \mathrm{~min}$ to stop the reaction and stored at $-80 \mathrm{C}$ until used in the PCR reaction.

For each PCR target, $5 \mu \mathrm{l}$ of the RT reaction product was amplified in duplicate using Fast Sybr Green mastermix (Applied Biosystems). The following transcripts were targeted: 18S ribosomal RNA (18S rRNA), caveolin 3 (CAV3), dysferlin (DYSF), cytochrome-c oxidase subunit 1 (COXI), cytochrome-c oxidase 4 (COXIV), succinate dehydrogenase subunit $b$ (SDHB), peroxisome proliferatoractivated receptor $\gamma$-co-activator $1 \alpha(\mathrm{PGC}-1 \alpha)$, Annexin $\mathrm{V}$ (ANXV), and SERCA2A. PCR primers were designed using Primer-BLAST (http://www.ncbi.nlm.nih.gov/tools/ primer-blast/). Primer sequences and Genbank accession numbers for the transcripts are shown in Table 2. Amplification efficiency of the primers used was 92.7-102.0\%, and melting curve analysis demonstrated specific amplification. The range of cycle threshold values was $13-25$. For all transcripts, the $18 \mathrm{~S}$ rRNA cycle threshold was subtracted from the mean cycle threshold value of the specific target to obtain $\Delta C_{t}$ and converted into relative concentrations by $2^{-\Delta C t}$.

\section{Statistics}

Statistical analyses were carried out with STATISTICA for windows (V10.0, StatSoft, Inc. (2011), www.statsoft.com) or SPSS16 (SPSS Inc, IL, USA). One sample was identified as an outlier in the RT-PCR experiments based on the Grubbs test and excluded from the further analysis. Immunofluorescence data from fibres demonstrating

\section{Table 2 Primers sequences used for RT-PCR analysis of} mRNA targets

\begin{tabular}{lll}
\hline $\begin{array}{l}\text { mRNA target } \\
\text { mRNA }\end{array}$ & PCR primer sequence $\mathbf{5} \rightarrow \mathbf{3}$ & Genbank \\
\hline 18S RNA & Forward: CGAACGTCTGCCCTATCAACTT & EU 139318.1 \\
& Reverse: ACCCGTGGTCACCATGGTA & \\
COXI & Forward: TGCCAGTATTAGCAGCAGGT & X14848.1 \\
& Reverse: GAATTGGGTCTCCACCTCCA & \\
COXIV & Forward: AGTCCAATTGTACCGCATCC & NM 017202.1 \\
& Reverse: ACTCATTGGTGCCCTGTTC & \\
SDHB & Forward: CAGAGAAGGGATCTGTGGCT & NM 001100539.1 \\
& Reverse: TGTTGCCTCCGTGATGTTC & \\
PGC-1a & Forward: ATGAGAAGCGGGAGTCTGAA & NM 031347.1 \\
& Reverse: GCGCTCTTCAATTGCTTCT & \\
SERCA2A & Forward: GGCCCGAACTACCTGGAGCC & NM 001110139 \\
& Reverse: CAACGCACATGCACGCACCC & \\
CAV3 & Forward: CCCAAGAACATCAATGAGGAC & U31968 \\
& Reverse: GGAGACGGTGAAAGTGGTT & \\
DYSF & Forward: TGGGAACCGCTACCATCTAC & NM001107869 \\
& Reverse: CTCTGGTGCAGGAAGGAGAC & \\
\hline
\end{tabular}


elevated or normal CaMKII levels in the same $\alpha / \beta$ CaMKII-transfected muscle were analysed with two-tailed $\mathrm{t}$-tests (unpaired). The fractions of MHCII-positive muscle fibres in the $\alpha / \beta$ CaMKII-transfected and non-transfected fibre pool, respectively, were analysed with Chi-squared tests because they represent categorical values. Western blot/immunodetection data from control-transfected and $\alpha / \beta$ CaMKII-transfected muscles were analysed with two-tailed Wilcoxon signed ranks tests. The effect of transfection on twitch and tetanic force parameters from $\alpha / \beta \quad$ CaMKII-transfected and control-transfected muscles was tested by relating force values to the respective mean of measures from the respective non-transfected muscles (i.e. GM and SOL) and running an ANOVA for the factors muscle (SOL, GM) $\mathrm{x}$ transfection (CaMKII-transfected, control-transfected). Non-parametric tests were run with exact significance. Significance level was set at $\mathrm{p}<0.05$. Values are provided as mean standard error (SE).

\footnotetext{
Abbreviations

18 rRNA: 18 S ribosomal RNA; ANXV: Annexin V; ATP5A: ATP synthase $\mathrm{H}+$ transporting mitochondrial F1 complex; CaMKII: Calcium/calmodulindependent protein kinase II; CAV3: Caveolin 3; COXI: Cytochrome-c oxidase subunit 1; COXIV: Cytochrome-c oxidase subunit 4; CaM: Calmodulin; DYSF: Dysferlin; EGTA: Ethylene glycol-bis(2-aminoethylether)-tetraacetic acid; Ftwitch: Maximum twitch force; Fmax: Maximum tetanic force; GM: Musculus gastrocnemius; HRT: Half-relaxation time; MHCIIA: Type IIA myosin heavy chain; MHCIIB: Type IIB myosin heavy chain; MHCIIX: Type IIX myosin heavy chain; myoD: Myogenic factor 3; NDUFA9: NADH dehydrogenase (ubiquinone) 1 alpha subcomplex 9; NTf: Non-transfected; PGC-1a: Peroxisome proliferator-activated receptor $\gamma$-co-activator $1 \mathrm{a}$; PCR: Polymerase chain reaction; pThr287-CaMKII: Threonine 287 phosphorylated CaMKII; SERCA2: Sarco/endoplasmic reticulum Ca2 + -ATPase; SDHA: Succinate dehydrogenase subunit b; SDHB: Succinate dehydrogenase subunit b; SE: Standard error; skaa: Skeletal alpha actin; SOL: Musculus soleus; SR: Sarcoplasmic reticulum; Tf: Transfected; TTP: Time-to-peak-twitch force; UQCRC2: Ubiquinol-cytochrome c reductase core II protein.
}

\section{Competing interests}

The authors declare that no academic, financial or non-financial competing interest exists.

\section{Authors contributions}

Conception and design of research: WE, RTJ, ADH, MF; Performed experiments: WE, CF, PV, MF; Analyzed data: WE, MF, RTJ; Interpreted results of experiments: WE, RTJ, MF; Funding: ADH, MF; Prepared figures: WE, MF; Drafted manuscript: WE, MF; Edited and revised manuscript: WE, RTJ, ADH, MF. All authors read and approved the final version of manuscript.

\section{Authors information}

WE carried out his work during the course of his PhD project under supervision of MF, RJ and AdH. He is currently a postdoctoral fellow involved in developing gene therapies for muscular dystrophies and type 2 diabetes. $\mathrm{RJ}$ is assistant professor and head of the Laboratory for Myology of the MOVE Research Institute Amsterdam. His research interest is in molecular physiology with a focus on mechano-transduction and adaptation of muscle size and oxidative capacity. Basic insights are translated into research on improvement of muscle function in sports, neuromuscular disorders and aging. AdH is Professor in Exercise Physiology at the Faculty of Human Movement Sciences, VU University Amsterdam, the Netherlands and Director of MOVE Research Institute Amsterdam, the Netherlands. His main professional interests concern short-term changes in metabolic and functional muscle characteristics (fatigue, potentiation) and adaptations to increased and decreased muscle activity as a result of training, disease, bedrest, spinal cord injury and aging. With integrative and translational research, he tries to bridge the gap between research at the genetic/molecular level and research performed at the whole human body level.

$\mathrm{CF}$ is a research assistant who contributed to critical experimentation during the revision of the manuscript.

PV is a postdoctoral fellow assessing molecular pathways of muscle plasticity who provided substantial input on the control experiments.

MF is Professor of Muscle Plasticity at the Orthopedics Clinics of the University of Zurich at Balgrist. His research centers on the signaling pathways that integrate muscle plasticity in response to use-related and clinical forms of stimuli. Towards this end he deploys an integrative approach combining the use of modern omics platforms, gene technology and classical physiological measures in rodent and human models. He is in the editorial board of The European Journal of Applied Physiology, Physiological Genomics and BMC Physiology.

\section{Acknowledgements}

We thank Carla Offringa for carrying out the RT-PCR experiments and Guus C. Baan for assistance with the muscle contraction measures, Dr. M. Neal Waxham (The University of Texas Medical School, Houston, USA) for donating the $a / \beta$-CaMKII plasmids, Dr. Frank W. Booth (University of Missouri,

Columbia, USA) for donating the skeletal alpha-actin reporter plasmid and Dr. Marie-Nolle Giraud (University of Bern, Bern, Switzerland) for donating the SERCA2 antibodies.

The study was supported through start-up grants from Manchester Metropolitan University (Manchester, United Kingdom), and VU University Amsterdam (Amsterdam, the Netherlands Kingdom), and grant

310000-112139 from the Swiss National Science Foundation.

\section{Author details}

${ }^{1}$ Institute for Biomedical Research into Human Movement and Health, Manchester Metropolitan University, John Dalton Building, Oxford Road, M1 5GD Manchester, United Kingdom. 'Laboratory for Myology, MOVE Research Institute Amsterdam, Faculty of Human Movement Sciences, VU University Amsterdam, Van der Boechorststraat 7, 1081 BT Amsterdam, The Netherlands. ${ }^{3}$ Laboratory for Muscle Plasticity, Department of Orthopaedics, University of Zurich, Balgrist University Hospital, Forchstrasse 340, 8008 Zurich, Switzerland.

Received: 7 April 2014 Accepted: 23 October 2014

Published online: 17 December 2014

\section{References}

1. Berchtold MW, Brinkmeier H, Muntener M: Calcium ion in skeletal muscle: its crucial role for muscle function, plasticity, and disease. Physiol Rev 2000, 80:1215 1265 .

2. Chin ER: Role of $\mathrm{Ca} 2+/$ calmodulin-dependent kinases in skeletal muscle plasticity. J Appl Physiol 2005, 99:414 423.

3. Fluck M, Hoppeler $\mathrm{H}$ : Molecular basis of skeletal muscle plasticity from gene to form and function. Rev Physiol Biochem Pharmacol 2003, 146:159 216.

4. Darveau CA, Suarez RK, Andrews RD, Hochachka PW: Allometric cascade as a unifying principle of body mass effects on metabolism. Nature 2002, 417:166 170.

5. Daz-Herrera P, Torres A, Morcuende JA, Garca-Castellano JM, Calbet JA Sarrat R: Effect of endurance running on cardiac and skeletal muscle in rats. Histol Histopathol 2001, 16:29 35.

6. Wada M, Inashima S, Yamada T, Matsunaga S: Endurance training-induced changes in alkali light chain patterns in type IIB fibers of the rat. J App/ Physiol 2003, 4:923 9.

7. Kubis HP, Haller EA, Wetzel P, Gros G: Adult fast myosin pattern and $\mathrm{Ca} 2+$-induced slow myosin pattern in primary skeletal muscle culture. Proc Natl Acad Sci U S A 1997, 94:4205 4210.

8. Sreter FA, Lopez JR, Alamo L, Mabuchi K, Gergely J: Changes in intracellular ionized Ca concentration associated with muscle fiber type transformation. Am J Physiol 1987, 253:C296 C300.

9. Wright DC, Geiger PC, Han DH, Jones TE, Holloszy JO: Calcium induces increases in peroxisome proliferator-activated receptor gamma coactivator-1alpha and mitochondrial biogenesis by a pathway leading to p38 mitogen-activated protein kinase activation. J Biol Chem 2007, 282:18793 18799 . 
10. Tavi $\mathrm{P}$, Westerblad $\mathrm{H}$ : The role of in vivo $\mathrm{Ca}(2)(+)$ signals acting on $\mathrm{Ca}(2)$ (+)-calmodulin-dependent proteins for skeletal muscle plasticity. J Physiol 2011, 589:5021 5031.

11. Chin ER, Olson EN, Richardson JA, Yang Q, Humphries C, Shelton JM, Wu H, Zhu W, Bassel-Duby R, Williams RS: A calcineurin-dependent transcriptional pathway controls skeletal muscle fiber type. Genes Dev 1998, 12:2499 2509 .

12. Parsons SA, Millay DP, Wilkins BJ, Bueno OF, Tsika GL, Neilson JR, Liberatore CM, Yutzey KE, Crabtree GR, Tsika RW, Molkentin JD: Genetic loss of calcineurin blocks mechanical overload-induced skeletal muscle fiber type switching but not hypertrophy. J Bio/ Chem 2004, 279:26192 26200.

13. Serrano AL, Murgia M, Pallafacchina G, Calabria E, Coniglio P, Lomo T, Schiaffino S: Calcineurin controls nerve activity-dependent specification of slow skeletal muscle fibers but not muscle growth. Proc Natl Acad Sci US A 2001, 98:13108 13113.

14. Garcia-Roves PM, Huss J, Holloszy JO: Role of calcineurin in exercise-induced mitochondrial biogenesis. Am J Physiol Endocrinol Metabol 2006, 290:E1172 E1179.

15. Jiang LQ, Garcia-Roves PM, De Castro BT, Zierath JR: Constitutively active calcineurin in skeletal muscle increases endurance performance and mitochondrial respiratory capacity. Am J Physiol Endocrinol Metabol 2010, 298:E8 E16.

16. Long YC, Glund S, Garcia-Roves PM, ZIERATH JR: Calcineurin regulates skeletal muscle metabolism via coordinated changes in gene expression. J Biol Chem 2007, 282:1607 1614

17. Ojuka EO, Jones TE, Han DH, Chen M, Holloszy JO: Raising Ca2+ in L6 myotubes mimics effects of exercise on mitochondrial biogenesis in muscle. FASEB J 2003, 17:675 681

18. Wu H, Kanatous SB, Thurmond FA, Gallardo T, Isotani E, Bassel-Duby R, Williams RS: Regulation of mitochondrial biogenesis in skeletal muscle by CaMK. Science 2002, 296:349 352

19. Allen $\mathrm{DL}$, Leinwand $\mathrm{LA}$ : Intracellular calcium and myosin isoform transitions. Calcineurin and calcium-calmodulin kinase pathways regulate preferential activation of the lla myosin heavy chain promoter. J Biol Chem 2002, 277:45323 45330.

20. Mu X, Brown LD, Liu Y, Schneider MF: Roles of the calcineurin and CaMK signaling pathways in fast-to-slow fiber type transformation of cultured adult mouse skeletal muscle fibers. Physiol Genomics 2007, 30:300 312.

21. Hawkins C, Xu A, Narayanan N: Sarcoplasmic reticulum calcium pump in cardiac and slow twitch skeletal muscle but not fast twitch skeletal muscle undergoes phosphorylation by endogenous and exogenous Ca2+/calmodulin-dependent protein kinase. Characterization of optimal conditions for calcium pump phosphorylation. J Biol Chem 1994, 269:31198 31206.

22. Tavi P, Allen DG, Niemela P, Vuolteenaho O, Weckstrom M, Westerblad H: Calmodulin kinase modulates $\mathrm{Ca}+$ release in mouse skeletal muscle. J Physiol 2003, 551:5 12.

23. Akimoto T, Ribar TJ, Williams RS, Yan Z: Skeletal muscle adaptation in response to voluntary running in $\mathrm{Ca} 2+$ /calmodulin-dependent protein kinase IV-deficient mice. Am J Physiol Cell Physiol 2004, 287:C1311 C1319.

24. Witczak CA, Jessen N, Warro DM, Toyoda T, Fujii N, Anderson ME, Hirshman MF Goodyear ᄂ: CaMKII regulates contraction- but not insulin-induced glucose uptake in mouse skeletal muscle. Am J Physiol Endocrinol Metabol 2010, 298:E1150 E1160.

25. Anderson ME, Brown JH, Bers DM: CaMKII in myocardial hypertrophy and heart failure. J Mol Cell Cardiol 2011, 51:468 473.

26. De Koninck $\mathrm{P}$, Schulman $\mathrm{H}$ : Sensitivity of CaM kinase II to the frequency of Ca2+ oscillations. Science 1998, 279:227 230.

27. Rose AJ, Alsted TJ, Kobbero JB, Richter EA: Regulation and function of Ca2 + -calmodulin-dependent protein kinase II of fast-twitch rat skeletal muscle. J Physio/ 2007, 580:993 1005

28. Rose AJ, Kiens B, Richter EA: Ca2 + -calmodulin-dependent protein kinase expression and signalling in skeletal muscle during exercise. J Physiol 2006, 574:889 903 .

29. Eilers W, Gevers W, Van Overbeek D, De Haan A, Jaspers RT, Hilbers PA, Van Riel N, Fluck M: Muscle-Type Specific Autophosphorylation of CaMKII Isoforms after Paced Contractions. BioMed Res Internat 2014, 943806. doi:10.1155/2014/943806. Epub 2014 Jun 26

30. Jain SS, Paglialunga S, Vigna C, Ludzki A, Herbst EA, Lally JS, Schrauwen P, Hoeks J, Tupling AR, Bonen A, Holloway GP: High-fat diet-induced mitochondrial biogenesis is regulated by mitochondrial derived reactive oxygen species activation of CaMKII. Diabetes 2014, 63(6):1907 1913.
31. Benziane B, Burton TJ, Scanlan B, Galuska D, Canny BJ, Chibalin AV, Zierath JR, Stepto NK: Divergent cell signaling after short-term intensified endurance training in human skeletal muscle. Am J Physiol Endocrinol Metab 2008, 295:E1427 E1438.

32. Rose AJ, Frosig C, Kiens B, Wojtaszewski JF, Richter EA: Effect of endurance exercise training on $\mathrm{Ca} 2+$ calmodulin-dependent protein kinase II expression and signalling in skeletal muscle of humans. J Physiol 2007, 583:785 795.

33. Baylor SM, Hollingworth S: Sarcoplasmic reticulum calcium release compared in slow-twitch and fast-twitch fibres of mouse muscle. J Physiol 2003, 551:125 138.

34. Hennig R, Lomo T: Firing patterns of motor units in normal rats. Nature 1985, 314:164 166.

35. Gaertner TR, Kolodziej SJ, Wang D, Kobayashi R, Koomen JM, Stoops JK, Waxham MN: Comparative analyses of the three-dimensional structures and enzymatic properties of alpha, beta, gamma and delta isoforms of Ca2 + -calmodulin-dependent protein kinase II. J Biol Chem 2004, 279:12484 12494.

36. Woodgett JR, Cohen P, Yamauchi T, Fujisawa H: Comparison of calmodulin-dependent glycogen synthase kinase from skeletal muscle and calmodulin-dependent protein kinase-II from brain. FEBS Lett 1984, 170:49 54.

37. Gorassini M, Eken T, Bennett DJ, Kiehn O, Hultborn H: Activity of hindlimb motor units during locomotion in the conscious rat. J Neurophysio/ 2000 83:2002 2011.

38. Durieux AC, Bonnefoy R, Manissolle C, Freyssenet D: High-efficiency gene electrotransfer into skeletal muscle: description and physiological applicability of a new pulse generator. Biochem Biophys Res Commun 2002, 296:443 450.

39. Durieux AC, D'antona G, Desplanches D, Freyssenet D, Klossner S, Bottinelli R, Fluck M: Focal adhesion kinase is a load-dependent governor of the slow contractile and oxidative muscle phenotype. J Physiol 2009, 587:3703 3717.

40. Rizzuto G, Cappelletti M, Maione D, Savino R, Lazzaro D, Costa P, Mathiesen I, Cortese R, Ciliberto G, Laufer R, La Monica N, Fattori E: Efficient and regulated erythropoietin production by naked DNA injection and muscle electroporation. Proc Natl Acad Sci U S A 1999, 96:6417 6422

41. Bayer $K$, Harbers $K$, Schulman H: alphaKAP is an anchoring protein for a novel CaM kinase II isoform in skeletal muscle. EMBO J 1998 , 17:5598 5605

42. Duguez S, Feasson L, Denis C, Freyssenet D: Mitochondrial biogenesis during skeletal muscle regeneration. Am J Physiol Endocrinol Metabol 2002, 282:E802 E809.

43. Shu B, Shen Y, Wang AM, Fang XQ, Li X, Deng HY, Yu ZQ: Histological, enzymohistochemical and biomechanical observation of skeletal muscle injury in rabbits. Chin J Traumatol 2007, 10:150 153.

44. Esposito A, Germinario E, Zanin M, Palade PT, Betto R, Danieli-Betto D: Isoform switching in myofibrillar and excitation-contraction coupling proteins contributes to diminished contractile function in regenerating rat soleus muscle. J Appl Physiol 2007, 102:1640 1648.

45. Fluck M, Schmutz S, Wittwer M, Hoppeler H, Desplanches D: Transcriptional reprogramming during reloading of atrophied rat soleus muscle. Am J Physiology Regul Integ Comp Physiol 2005, 289:R4 R14.

46. Wang J, Best PM: Inactivation of the sarcoplasmic reticulum calcium channel by protein kinase. Nature 1992, 359:739 741.

47. Dulhunty AF, Laver D, Curtis SM, Pace S, Haarmann C, Gallant EM: Characteristics of irreversible ATP activation suggest that native skeletal ryanodine receptors can be phosphorylated via an endogenous CaMKII. Biophysical J 2001, 81:3240 3252.

48. Xu A, Narayanan N: Ca2+/calmodulin-dependent phosphorylation of the Ca2 + -ATPase, uncoupled from phospholamban, stimulates Ca2 + -pumping in native cardiac sarcoplasmic reticulum. Biochem Biophys Res Commun 1999, 258:66 72.

49. Arcuri C, Giambanco I, Bianchi R, Donato R: Annexin V, annexin Vl, S100A1 and $\mathrm{S} 100 \mathrm{~B}$ in developing and adult avian skeletal muscles. Neuroscience 2002, 109:371 388.

50. Zador E, Mendler L, Ver Heyen M, Dux L, Wuytack F: Changes in mRNA levels of the sarcoplasmic/endoplasmic-reticulum $\mathrm{Ca}(2+)$-ATPase isoforms in the rat soleus muscle regenerating from notexin-induced necrosis. Biochem J 1996, 320(Pt 1):107 113.

51. Ramirez MT, Zhao XL, Schulman $\mathrm{H}$, Brown $\mathrm{JH}$ : The nuclear deltaB isoform of $\mathrm{Ca} 2+/$ calmodulin-dependent protein kinase II regulates atrial 
natriuretic factor gene expression in ventricular myocytes. $J$ Biol Chem 1997, 272:31203 31208.

52. Ronkainen JJ, Hanninen SL, Korhonen T, Koivumaki JT, Skoumal R, Rautio S, Ronkainen VP, Tavi P: Ca2 + -calmodulin-dependent protein kinase II represses cardiac transcription of the L-type calcium channel alpha(1C)-subunit gene (Cacna1c) by DREAM translocation. J Physiol 2011, 589:2669 2686.

53. Srinivasan $\mathrm{M}$, Edman CF, Schulman $\mathrm{H}$ : Alternative splicing introduces a nuclear localization signal that targets multifunctional $\mathrm{CaM}$ kinase to the nucleus. J Cell Biol 1994, 126:839 852

54. Sun P, Enslen H, Myung PS, Maurer RA: Differential activation of CREB by Ca2+/calmodulin-dependent protein kinases type II and type IV involves phosphorylation of a site that negatively regulates activity. Genes Dev 1994, 8:2527 2539.

55. Sun P, Lou L, Maurer RA: Regulation of activating transcription factor-1 and the cAMP response element-binding protein by $\mathrm{Ca} 2+/$ calmodulin-dependent protein kinases type I, II, and IV. J Biol Chem 1996, 271:3066 3073.

56. Murgia M, Elbenhardt Jensen T, Cusinato M, Garcia M, Richter EA, Schiaffino S: Multiple signalling pathways redundantly control GLUT4 gene transcription in skeletal muscle. J Physiol 2009, 587(Pt 17):4319 4327.

57. Klossner S, Dapp C, Schmutz S, Vogt M, Hoppeler H, Fluck M: Muscle transcriptome adaptations with mild eccentric ergometer exercise. Pflugers Arch 2007, 455:555 562.

58. Schmutz S, Dapp C, Wittwer M, Vogt M, Hoppeler H, Fluck M: Endurance training modulates the muscular transcriptome response to acute exercise. Pflugers Arch 2006, 451:678 687.

59. Marsh DR, Carson JA, Stewart LN, Booth FW: Activation of the skeletal alpha-actin promoter during muscle regeneration. J Muscle Res Cell Motil 1998, 19:897 907.

60. Haan A, Huijing PA, Vliet MR: Rat medial gastrocnemius muscles produce maximal power at a length lower than the isometric optimum length. Pflugers Archiv Europ J Physiol 2003, 445:728 733.

61. De Haan A, De Ruiter CJ, Lind A, Sargeant AJ: Age-related changes in force and efficiency in rat skeletal muscle. Acta Physiol Scand 1993, 147:347 355.

62. Fluck M, Waxham MN, Hamilton MT, Booth FW: Skeletal muscle $\mathrm{Ca}(2+)$-independent kinase activity increases during either hypertrophy or running. J Appl Physiol 2000, 88:352 358.

63. Desplanches D, Amami M, Dupr-Aucouturier S, Valdivieso P, Schmutz S, Mueller M, Hoppeler H, Kreis R, Flck M: Hypoxia refines plasticity of mitochondrial respiration to repeated muscle work. Eur J Appl Physiol 2014, 114(2):405 417.

64. Van Wessel T, De Haan A, van der Laarse WJ, Jaspers RT: The muscle fiber type-fiber size paradox: hypertrophy or oxidative metabolism? Eur J App/ Physiol 2010, 110:665 694.

doi:10.1186/s12899-014-0007-z

Cite this article as: Eilers et al: CaMKII content affects contractile, but not mitochondrial, characteristics in regenerating skeletal muscle. $B M C$ Physiology 2014 14:7.

\section{Submit your next manuscript to BioMed Central and take full advantage of:}

$\otimes$ Convenient online submission

$\otimes$ Thorough peer review

$\otimes$ No space constraints or color $\nabla$ gure charges

$\triangle$ Immediate publication on acceptance

Q Inclusion in PubMed, CAS, Scopus and Google Scholar

$\otimes$ Research which is freely available for redistribution 